\title{
Anti-periodic Boundary Value Problems of $\varphi$-Laplacian Impulsive Differential Equations
}

\author{
Xiufeng Guo*, Yuan Gu \\ College of Sciences, Hezhou University, Hezhou, Guangxi, China \\ Email address: \\ lgxf88@163.com (Xiufeng Guo), 781349068@qq.com (Yuan Gu) \\ ${ }^{*}$ Corresponding author
}

To cite this article:

Xiufeng Guo, Yuan Gu. Anti-periodic Boundary Value Problems of $\varphi$-Laplacian Impulsive Differential Equations. Applied and Computational Mathematics. Vol. 5, No. 2, 2016, pp. 91-96. doi: 10.11648/j.acm.20160502.19

Received: May 3, 2016; Accepted: May 13, 2016; Published: May 30, 2016

\begin{abstract}
This paper concerned with the existence of solutions of anti-periodic boundary value problems for impulsive differential equations with $\varphi$-Laplacian operator. Firstly, the definition a pair of coupled lower and upper solutions of the problem is introduced. Then, under the approach of coupled upper and lower solutions together with Nagumo condition, we prove that there exists at least one solution of anti-periodic boundary value problems for impulsive differential equations with $\varphi$-Laplacian operator.
\end{abstract}

Keywords: Anti-periodic Boundary Value Problems, Impulsive Differential Equations, $\varphi$-Laplacian Operator, Coupled Lower and Upper Solutions

\section{Introduction}

In this paper, we will study a class of nonlinear impulsive differential equation with $\varphi$-Laplacian operator and anti-periodic boundary conditions as following:

$$
\begin{gathered}
\left(\varphi\left(u^{\prime}(t)\right)\right)^{\prime}=f\left(t, u(t), u^{\prime}(t)\right) \quad \text { a.e. } t \in[0, T], P, \\
\left\{\begin{array}{c}
I_{k}\left(u\left(t_{k}\right), u\left(t_{k}^{+}\right)\right)=0, \\
M_{k}\left(u\left(t_{k}\right), u\left(t_{k}^{+}\right), u^{\prime}\left(t_{k}\right), u^{\prime}\left(t_{k}^{+}\right), u\right)=0,
\end{array}\right. \\
u(0)=-u(T), u^{\prime}(0)=-u^{\prime}(T), 2, \cdots, p,
\end{gathered}
$$

where $\varphi$ is an increasing homeomorphism from $R$ to $R$, and $f:[0, T] \times R^{2} \rightarrow R \quad$ is a Carathéodory function. $P=\left\{t_{1}, \cdots, t_{p}: 0=t_{0}<t_{1}<\cdots<t_{p}<t_{p+1}=T\right\}, I_{k} \in C^{0}\left(R^{2}\right)$, $M_{k} \in C^{0}\left(R^{4} \times C_{P}^{1}\right), k=1, \cdots, p$ are impulsive functions and $C_{P}^{1}$ will be given later.

Impulsive differential equations have become an important aspect in some mathematical models of real processes and phenomena in science. There has a significant development in impulse theory and impulsive differential equations (see $[1,2$, $3]$ ). Moreover, $p$-Laplacian operator arises in non-Newtonian fluid flows, turbulent filtration in porous media and in many other application areas (see $[5,7]$ and references therein). Usually, $p$-Laplacian operator is replaced by abstract and more general version $\varphi$-Laplacian operator, which lead to clearer expositions and a better understanding of the methods which ware employed to derive the existence results (see [12, 13]). Recently, Cabada and Tomecek [4] focussed on the $\varphi$-Laplacian differential equations (1) subject to impulsive functions (2) with non-local boundary conditions

$$
\left\{\begin{array}{c}
g_{1}(u(0), u(T))=0, \\
g_{2}\left(u(0), u(T), u^{\prime}(0), u^{\prime}(T), u\right)=0,
\end{array}\right.
$$

Later, the paper [6] generalize the problem of [4] and considered a more general $\varphi$-Laplacian differential equations

$$
-\frac{d}{d t}\left(\varphi\left(t, u, u(t), u^{\prime}(t)\right)\right)=f\left(t, u, u(t), u^{\prime}(t)\right), \text { a.e. } t \in[0, T], \quad P
$$

coupled with the compatible impulses and boundary conditions

$$
\left\{\begin{array}{c}
G_{0}\left(u(0), u(T), u, u^{\prime}(0), u^{\prime}(T)\right)=(0,0), \\
G_{k}\left(u\left(t_{k}^{+}\right), u\left(t_{k}^{-}\right), u, u^{\prime}\left(t_{k}^{+}\right), u^{\prime}\left(t_{k}^{-}\right)\right)=(0,0), k=1,2, \cdots, p,
\end{array}\right.
$$


where $\varphi \in C\left([0, T] \times C_{P}^{1} \times R^{2}\right)$, and $f:[0, T] \times C_{P}^{1} \times R^{2} \rightarrow R$ is a Carathéodory function, $G_{k}=\left(g_{k, 1}, g_{k, 2}\right)$ $\in C\left(R^{2} \times C_{P}^{1} \times R^{2} ; R^{2}\right), k=0, \cdots, p$. After make a study of the monotonicity of the boundary condition functions in $[4,6$, $8]$, we found that their boundary problems didn't include the anti-periodic boundary condition problems.

Motivated by above the mentioned papers, we try to find some appropriate conditions to make sure the existence solutions for anti-periodic problem (1)-(3). As far as we known, although the papers $[4,6,8]$ study such a general $\varphi$-Laplacian problems with nonlinear boundary conditions, but its didn't contain the anti-periodic problems. Besides, there are few dependent references for studying the $\varphi$-Laplacian impulsive functional differential equations with anti-periodic boundary condition. Furthermore, the anti-periodic problem is a very important area of research. The study of anti-periodic problem for nonlinear evolution equations is closely related to the study of periodic problem which was initiated by Okochi [14]. Anti-periodic problem has been extensively studied during the past decades, such as anti-periodic trigonometric polynomials ([15]). Moreover, anti-periodic boundary conditions also appear in difference and differential equations (see $[16,17]$ and references therein).

This paper is organized as follows: In section 2, we state some preliminaries that will be used throughout the paper. In section 3, we obtain existence solutions for problem (1)-(3) by the approach of coupled upper and lower solutions together with Nagumo condition. Finally, we give the conclusion of our main work in section 4 .

\section{Preliminaries}

For a real valued measurable function $u$ defined almost everywhere on $I \subset R$ and $q \geq 1$, let

$$
\|u\|_{q}=\left(\int_{I}|u(s)|^{q} d s\right)^{\frac{1}{q}} \text { and }\|u\|_{\infty}=\sup _{t \in I} \operatorname{ess}|u(t)| .
$$

For a given Banachspace $E$, let $C^{0}(E)$ be the set of all continuous functions $f: E \rightarrow R$. Let $C^{m}(I)$ be the set of all functions $u$ which are $m$ times continuously differentiable on $I$ with finite norm

$$
\|u\|_{C^{m}(I)}=\max _{k=0, \cdots, m}\left\|u^{(k)}\right\|_{\infty} .
$$

For $1 \leq q \leq \infty, L^{q}(I)$ denotes the set of Lebesgue measurable functions $u$ on $I$ such that $\|u\|_{q}$ is finite. Let $A C(I, q)$ be the set of absolutely continuous functions $u$ on $I$ satisfy $u^{\prime} \in L^{q}(I) . W^{m, q}(I)$ denotes the set of functions $u \in C^{m-1}(I)$ and $u^{(m-1)} \in A C(I, q)$ with finite norm

$$
\|u\|_{W^{m, q}(I)}=\max _{k=0, \cdots, m}\left\|u^{(k)}\right\|_{q}
$$

It is well known that $C^{m}(I)$ and $W^{m, q}(I)$ are Banach spaces and $W^{m, q}(I)$ is a usual Sobolev space.

Let $p \in N$. A finite subset $P$ of the interval $[0, T]$ defined by

$$
P=\left\{t_{1}, \cdots, t_{p}: 0=t_{0}<t_{1}<\cdots<t_{p}<t_{p+1}=T\right\} .
$$

Let $J_{0}=\left[0, t_{1}\right]$ and $J_{k}=\left(t_{k}, t_{k+1}\right]$ for all $k=1, \cdots, p$. For $m \in N \cup\{0\}$ and $1 \leq q \leq \infty$, we denote

$$
C_{P}^{m}=\left\{u:[0, T] \rightarrow R: \text { for all } k=0, \cdots, p, u \in C^{m}\left(J_{k}\right),\right.
$$
there exist $u^{(l)}\left(t_{k}^{+}\right)$,

$$
\begin{aligned}
& \left.k=1, \cdots, p \text { and } u^{(l)}\left(t_{k}^{-}\right)=u^{(l)}\left(t_{k}\right), k=1, \cdots, p+1 ; l=0, \cdots, m\right\} \\
& W_{P}^{m, q}=\left\{u:[0, T] \rightarrow R: u_{J_{k}} \in W^{m, q}\left(J_{k}\right), k=0, \cdots, p\right\} .
\end{aligned}
$$

It is easy to verify that the spaces $C_{P}^{m}$ and $W_{P}^{m, q}$ are Banach spaces with the norms

$$
\|u\|_{C_{P}^{m}}=\max _{k=0, \cdots, p}\left\|u_{\mid J_{k}}\right\|_{C^{m}\left(J_{k}\right)} \quad \text { and }\|u\|_{W_{P}^{m, q}}=\max _{k=0, \cdots, p}\left\|u_{\mid J_{k}}\right\|_{W^{m, q}\left(J_{k}\right)} .
$$

We say that $f:[0, T] \times S \rightarrow R\left(S \subset R^{2}\right)$ satisfies the restricted Carathéodory conditions on $[0, T] \times S$ if

(i) for each $x \in S$ the function $f(\cdot, x)$ is measurable on $[0, T]$;

(ii) the function $f(t, \cdot)$ is continuous on $S$ a.e. $t \in[0, T]$;

(iii) for every compact set $K \subset S$, there exists a nonnegative function $\mu_{K} \in L^{1}(0, T)$ such that

$$
|f(t, x)| \leq \mu_{K}(t) \quad \text { for a.e. } t \in[0, T] \text { and all } x \in K \text {. }
$$

In this paper, we use $\operatorname{Car}([0, T] \times S)$ to denote the set of functions satisfying the restricted Carathéodory conditions on $[0, T] \times S$. In what follows, $D^{ \pm}$and $D_{ \pm}$denote the Dini derivatives.

Definition 1. The functions $\alpha, \beta \in W_{P}^{1, \infty}$ such that $\alpha \leq \beta$ are said to be a pair of coupled lower and upper solutions of problem (1)-(3) if $\alpha, \beta$ satisfy the following conditions:

(i) $D_{-} \alpha(t) \leq D^{+} \alpha(t)$ for all $t \in[0, T], P$. Moreover, if $\tau \in[0, T], P$ such that $D_{-} \alpha(\tau)=D^{+} \alpha(\tau)$, then there exists $\varepsilon>0 \quad$ such that $\quad \alpha \in C^{1}([\tau-\varepsilon, \tau+\varepsilon])$, $\varphi \circ \alpha^{\prime} \in A C([\tau, \tau+\varepsilon])$ and

$$
\left(\varphi\left(\alpha^{\prime}(t)\right)\right)^{\prime} \geq f\left(t, \alpha(t), \alpha^{\prime}(t)\right) \quad \text { a.e. } t \in[\tau, \tau+\varepsilon] .
$$

(ii) $D_{-} \beta(t) \geq D^{+} \beta(t)$ for all $t \in[0, T], P$. Moreover, if $\tau \in[0, T], P$ such that $D_{-} \beta(\tau)=D^{+} \beta(\tau)$, then there exists $\varepsilon>0 \quad$ such that $\quad \beta \in C^{1}([\tau-\varepsilon, \tau+\varepsilon])$, $\varphi \circ \beta^{\prime} \in A C([\tau, \tau+\varepsilon])$ and

$$
\left(\varphi\left(\beta^{\prime}(t)\right)\right)^{\prime} \leq f\left(t, \beta(t), \beta^{\prime}(t)\right) \text { a.e. } t \in[\tau, \tau+\varepsilon] .
$$

(iii) For all $k=1, \cdots, p, I_{k}\left(\alpha\left(t_{k}\right), \cdot\right)$ are injective and there exist $D^{+} \alpha\left(t_{k}\right), D_{-} \alpha\left(t_{k}\right), D_{+} \beta\left(t_{k}\right), D^{-} \beta\left(t_{k}\right) \in R$ such that 


$$
\begin{aligned}
& I_{k}\left(\alpha\left(t_{k}\right), \alpha\left(t_{k}^{+}\right)\right)=0 \leq M_{k}\left(\alpha\left(t_{k}\right), \alpha\left(t_{k}^{+}\right), D_{-} \alpha\left(t_{k}\right), D^{+} \alpha\left(t_{k}\right), \alpha\right), \\
& I_{k}\left(\beta\left(t_{k}\right), \beta\left(t_{k}^{+}\right)\right)=0 \geq M_{k}\left(\beta\left(t_{k}\right), \beta\left(t_{k}^{+}\right), D^{-} \beta\left(t_{k}\right), D_{+} \beta\left(t_{k}\right), \beta\right) .
\end{aligned}
$$

and there exist $D^{+} \alpha(0), D_{-} \alpha(T), D_{+} \beta(0), D^{-} \beta(T) \in R$ such that

$$
\begin{aligned}
& \alpha(0)+\beta(T)=0 \leq D^{+} \alpha(0)+D^{-} \beta(T), \\
& \alpha(T)+\beta(0)=0 \geq D_{-} \alpha(T)+D_{+} \beta(0) .
\end{aligned}
$$

Definition 2. Given a function $u \in C_{P}^{1}$ is called a solution of the problem (1)-(3) if $\varphi \circ u^{\prime} \in W_{P}^{1,1}$ and $u$ satisfies (1) and fulfills conditions (2) and (3).

Definition 3. Assume that the functions $f \in \operatorname{Car}\left([0, T] \times R^{2}\right)$ and $\alpha, \beta \in W_{P}^{1, \infty}$ satisfying $\alpha(t) \leq \beta(t)$ for $\forall t \in[0, T]$. We say that the function $f$ satisfies a Nagumo condition with respect to $\alpha$ and $\beta$ if, for $k=1, \cdots, p$, there exist functions $\phi_{k} \in C[0, \infty)$ and $w \in L^{q}(0, T), 1 \leq q \leq \infty$, such that $\phi_{k}>0$ on $[0, \infty)$,

\section{$|f(t, u, v)| \leq w(t) \phi_{k}(|v|) \quad$ on $J_{k} \times[\alpha(t), \beta(t)] \times R$.}

Moreover, there exists a constant $K=K(\alpha, \beta)$ with $K>\max \left\{r_{k},\left\|\alpha^{\prime}\right\|_{\infty},\left\|\beta^{\prime}\right\|_{\infty}\right\}$, such that the following inequalities

$$
\int_{\varphi\left(r_{k}\right)}^{\varphi\left(\left(\varphi^{\prime}\right)\right.} \frac{\left(\varphi^{-1}(x)\right)^{(q-1) / q} / q}{\phi_{k}\left(\varphi^{-1}(x)\right)} d x,-\int_{\varphi(-K)}^{\varphi\left(-r_{k}\right)} \frac{\left(\varphi^{-1}(x)\right)^{(q-1) / q}}{\phi_{k}\left(-\varphi^{-1}(x)\right)} d x>\|w\|_{J_{k}, q} \eta_{k}^{(q-1) / q}
$$

hold, where

$$
\eta_{k}=\sup _{t \in J_{k}} \beta(t)-\inf _{t \in J_{k}} \alpha(t)
$$

$r_{k}=\frac{1}{t_{k+1}-t_{k}} \max \left\{\beta\left(t_{k+1}^{-}\right)-\alpha\left(t_{k}^{+}\right), \beta\left(t_{k}^{+}\right)-\alpha\left(t_{k+1}^{-}\right)\right\}$. Moreover, any constant such $K>\max \left\{r_{k}: k=0, \cdots, p\right\}>0$ will be called a Nagumo constant.

Throughout this paper, we impose the following hypotheses:

$\left(\mathrm{H}_{1}\right)$ The function $\varphi: R \rightarrow R$ is a continuous and strictly increasing.

$\left(\mathrm{H}_{2}\right)$ The problem (1)-(3) has a pair of coupled lower and upper solutions $\alpha$ and $\beta$.

$\left(\mathrm{H}_{3}\right)$ The function $f \in \operatorname{Car}\left([0, T] \times R^{2}\right)$ and satisfies a Nagumo condition with respect to $\alpha$ and $\beta$.

$\left(\mathrm{H}_{4}\right)$ For $k=1, \cdots, p$, the functions $I_{k} \in C^{0}\left(R^{2}\right)$ are non-decreasing in the first variable, $M_{k} \in C^{0}\left(R^{4} \times C_{P}^{1}\right)$ are non-increasing in the third variable and non-decreasing in the fourth and fifth variables.

\section{Existence Results}

In this section, we will deduce that there exists at least one solution of the problem (1)-(3) lying between a pair of coupled lower and upper solutions.
Firstly, we state the following existence and uniqueness result for a problem with linear right-hand side.

Lemma 1. (Lemma 7 of [4]). Let $\tilde{f} \in L^{1}[0, T]$ and $A_{k}, B_{k} \in R$ for each $k=0, \cdots, p$. Suppose that $\bar{\varphi}: R \rightarrow R$ is a strictly increasing function satisfies $\bar{\varphi}(R)=R$. Then the non homogeneous impulsive Dirichlet problem

$$
\left\{\begin{array}{c}
\left(\bar{\varphi}\left(u^{\prime}(t)\right)\right)^{\prime}=\tilde{f}(t) \quad \text { a.e. } t \in[0, T], \\
u\left(t_{k}\right)=B_{k-1}, u\left(t_{k}^{+}\right)=A_{k}, \quad k=1,2, \cdots, p, \\
u(0)=A_{0}, u(T)=B_{p},
\end{array}\right.
$$

has a unique solution $u$, which can be written in the form

$$
u(t)=A_{k}+\int_{t_{k}}^{t} \bar{\varphi}^{-1}\left(\int_{t_{k}}^{z} \tilde{f}(s) d s+\tau_{k}\right) d z, t \in J_{k}, k=0, \cdots, p,
$$

where $\tau_{k}$ is the unique solution of the equation

$$
B_{k}-A_{k}=\int_{t_{k}}^{t_{k+1}} \bar{\varphi}^{-1}\left(\int_{t_{k}}^{z} \tilde{f}(s) d s+\tau_{k}\right) d z .
$$

Next, let us consider the following functions

$$
\delta_{K}(y)=\min \{K, \max \{y,-K\}\} \text { for all } y \in R,
$$

where $K$ is the constant introduced in definition 2.3,

$$
\rho(t, u)=\min \{\beta(t), \max \{u, \alpha(t)\}\} \text { for }(t, u) \in[0, T] \times R,
$$

coupled with functionals $A_{k}, B_{k}: C_{P}^{1} \rightarrow R$ given by

$$
\begin{aligned}
& A_{0}(u)=\rho(0,-u(T)), \quad B_{p}(u)=\rho\left(T, u(T)-u^{\prime}(0)-u^{\prime}(T)\right), \\
& A_{k}(u)=\rho\left(t_{k}^{+}, u\left(t_{k}^{+}\right)+I_{k}\left(u\left(t_{k}\right), u\left(t_{k}^{+}\right)\right)\right), k=1, \cdots, p, \\
& B_{k-1}(u)=\rho\left(t_{k}, u\left(t_{k}\right)+M_{k}\left(u\left(t_{k}\right), u\left(t_{k}^{+}\right), u^{\prime}\left(t_{k}\right), u^{\prime}\left(t_{k}^{+}\right), u\right)\right), k=1, \cdots, p .
\end{aligned}
$$

Moreover, for each $u \in C_{P}^{1}$ we consider a function $\tilde{f}_{u}:[0, T] \rightarrow R$ defined by

$$
\tilde{f}_{u}(t)=f\left(t, \rho(t, u(t)), \delta_{K}\left(\frac{d}{d t} \rho(t, u(t))\right)\right) .
$$

The function $\tilde{f}_{u}$ is well defined according to the following result (by redefining function $\frac{d}{d t} \rho(t, u(t))$ as zero when it does not exist). It can be proved in a similar way to Lemma 2 in [10].

Lemma 2. For given $u, u_{n} \in C_{P}^{1}$ such that $u_{n} \rightarrow u$ in $C_{P}^{1}$, then

(i) $\frac{d}{d t} \rho(t, u(t))$ exists for a.e. $t \in[0, T], P$;

(ii) $\frac{d}{d t} \rho\left(t, u_{n}(t)\right) \rightarrow \frac{d}{d t} \rho(t, u(t))$ for a.e. $t \in[0, T], P$.

Now, define a strictly increasing homeomorphism $\bar{\varphi}: R \rightarrow R$ by: 


$$
x \in R \rightarrow \bar{\varphi}(x)=\left\{\begin{array}{c}
\varphi(x), \quad|x| \leq K, \\
\frac{\varphi(K)-\varphi(-K)}{2 K} x-\frac{1}{2}(\varphi(K)+\varphi(-K)),|x|>K .
\end{array}\right.
$$

In what follows, we are in a position to prove the existence theorem.

Theorem 3. Assume that the hypotheses $\left(\mathrm{H}_{1}\right)-\left(\mathrm{H}_{4}\right)$ hold. Then there exists at least one solution $u$ of the problem (1)-(3) such that

$$
\alpha(t) \leq u(t) \leq \beta(t) \quad \text { and } \quad\left|u^{\prime}(t)\right| \leq K, t \in[0, T],
$$

where $K=K(\alpha, \beta)$ is the constant introduced in Definition 2.3 .

Proof. First, we consider the following auxiliary problem

$$
\left(P^{*}\right)\left\{\begin{array}{c}
\left(\bar{\varphi}\left(u^{\prime}(t)\right)\right)^{\prime}=\tilde{f}_{u}(t), \text { a.e. } t \in[0, T], P, \\
u\left(t_{k}\right)=B_{k-1}(u), u\left(t_{k}^{+}\right)=A_{k}(u), k=1,2, \cdots, m, \\
u(0)=A_{0}(u), u(T)=B_{p}(u) .
\end{array}\right.
$$

From the definitions of functions $\rho$ and $\delta_{K}$, Lemma 3.2 and hypothesis $\left(\mathrm{H}_{3}\right)$, for $u \in C_{P}^{1}$, we have $\tilde{f}_{u} \in L^{1}([0, T])$. Define an operator $F: C_{P}^{1} \rightarrow C_{P}^{1}$ by

$$
F u(t)=A_{k}(u)+\int_{t_{k}}^{t} \bar{\varphi}^{-1}\left(\int_{t_{k}}^{z} \tilde{f}_{u}(s) d s+\tau_{k}(u)\right) d z, t \in J_{k}, k=0, \cdots, p
$$

for each $u \in C_{P}^{1}$, where $\tau_{k}(u)$ is given as the unique solution of equation

$$
B_{k}(u)-A_{k}(u)=\int_{t_{k}}^{t_{k+1}} \bar{\varphi}^{-1}\left(\int_{t_{k}}^{z} \tilde{f}_{u}(s) d s+\tau_{k}(u)\right) d z .
$$

The proof of the existence of a fixed point $u$ of operator $F$ follows in a similar way to the one given in Theorem 9 of [4]. In view of Lemma 3.1, we know that the fixed piont $u$ is a solution of the problem $\left(P^{*}\right)$.

Next, we prove that $\alpha \leq u \leq \beta$ on $[0, T]$.

Let $k \in\{0, \cdots, p\}$. From the definition of $\rho$, it follows that

$$
\alpha\left(t_{k}^{+}\right) \leq u\left(t_{k}^{+}\right), \alpha\left(t_{k+1}\right) \leq u\left(t_{k+1}\right)
$$

We will show that $u-\alpha$ is non-negative on $J_{k}$. Assume that there exists $\xi_{k} \in\left(t_{k}, t_{k+1}\right)$ such that

$$
\min _{t \in\left(t_{k}, t_{k+1}\right)}(u-\alpha)(t)=(u-\alpha)\left(\xi_{k}\right)<0,
$$

and there exists $\delta>0$ such that

$$
(u-\alpha)\left(\xi_{k}\right)<(u-\alpha)(t)<(u-\alpha)\left(\xi_{k}+\delta\right)=0 \quad \text { for all } t \in\left(\xi_{k}, \xi_{k}+\delta\right) .
$$

Then $D_{-}(u-\alpha)\left(\xi_{k}\right) \leq D^{+}(u-\alpha)\left(\xi_{k}\right)$. As $u \in C_{P}^{1}$, we have $D_{-} \alpha\left(\xi_{k}\right) \geq D^{+} \alpha\left(\xi_{k}\right)$. Together with the definition 2.1, there exists $\varepsilon>0$ such that $\alpha \in C^{1}\left(\left[\xi_{k}-\varepsilon, \xi_{k}+\varepsilon\right]\right)$. Thus we have that $(u-\alpha)^{\prime}\left(\xi_{k}\right)=0, \varphi \circ \alpha^{\prime} \in A C\left(\left[\xi_{k}, \xi_{k}+\varepsilon\right]\right)$. As a consequence, from the definition of $\alpha$ and $K$, we have

$$
\bar{\varphi}\left(u^{\prime}\left(\xi_{k}\right)\right)-\bar{\varphi}\left(\alpha^{\prime}\left(\xi_{k}\right)\right)=\varphi\left(u^{\prime}\left(\xi_{k}\right)\right)-\varphi\left(\alpha^{\prime}\left(\xi_{k}\right)\right)=0
$$

and

$$
\begin{aligned}
\bar{\varphi}\left(u^{\prime}(t)\right)-\bar{\varphi}\left(\alpha^{\prime}(t)\right) & =\bar{\varphi}\left(u^{\prime}(t)\right)-\varphi\left(\alpha^{\prime}(t)\right) \\
& =\int_{\xi_{k}}^{t}\left(\bar{\varphi}\left(u^{\prime}(s)\right)\right)^{\prime}-\left(\varphi\left(\alpha^{\prime}(s)\right)\right)^{\prime} d s \\
& \leq \int_{\xi_{k}}^{t}\left(\tilde{f}_{u}(s)-f\left(s, \alpha(s), \alpha^{\prime}(s)\right) d s=0,\right.
\end{aligned}
$$

for all $t \in\left(\xi_{k}, \xi_{k}+\varepsilon\right)$. In view of the monotonicity of $\bar{\varphi}$ we get $u^{\prime}(t) \leq \alpha^{\prime}(t), t \in\left(\xi_{k}, \xi_{k}+\varepsilon\right)$, which contradict to (5).

Similarly, we can prove that $\beta-u$ is non-negative on $[0, T]$.

Therefore, any solution of $\left(P^{*}\right)$ satisfies

$$
\left(\bar{\varphi}\left(u^{\prime}(t)\right)\right)^{\prime}=f\left(t, u(t), \delta_{K}\left(u^{\prime}(t)\right)\right) \text {, a.e. } t \in[0, T] .
$$

To see any solution $u$ of $\left(P^{*}\right)$ satisfies $\left|u^{\prime}(t)\right| \leq K$ for all $t \in[0, T]$. By contradiction, we assume

$$
\sup _{t \in J_{k}}\left|u^{\prime}(t)\right|>K
$$

Then there exists $\xi_{k} \in\left(t_{k}, t_{k+1}\right)$ such that

$$
\left|u^{\prime}\left(\xi_{k}\right)\right|=\frac{\left|u\left(t_{k+1}\right)-u\left(t_{k}^{+}\right)\right|}{t_{k+1}-t_{k}} \leq r_{k}
$$

Since $u \in C^{1}\left(J_{k}\right)$, we have $-K<-r_{k} \leq u^{\prime}\left(\xi_{k}\right) \leq r_{k}<K$, then there exists $\bar{t} \in J_{k}$ such that $u^{\prime}(\bar{t})>K$ or $u^{\prime}(\bar{t})<-K$. If $u^{\prime}(\bar{t})>K$, there exist $s_{1}, s_{2} \in J_{k}$ such that

$$
u^{\prime}\left(\xi_{k}\right) \leq r_{k}=u^{\prime}\left(s_{1}\right)<u^{\prime}(t)<u^{\prime}\left(s_{2}\right)=K<u^{\prime}(\bar{t}), t \in I,
$$

where $I=\left(s_{1}, s_{2}\right)$ or $I=\left(s_{2}, s_{1}\right)$. In what follows, we only consider the case $I=\left[s_{1}, s_{2}\right]$ since the other case can be treated similarly. By variable substitution, we have

$$
\begin{aligned}
\int_{\varphi\left(r_{k}\right)}^{\varphi(K)} \frac{\left(\varphi^{-1}(x)\right)^{(q-1) / q}}{\phi_{k}\left(\varphi^{-1}(x)\right)} d x & =\int_{\varphi\left(u^{\prime}\left(s_{1}\right)\right)}^{\varphi\left(u^{\prime}\left(s_{2}\right)\right)} \frac{\left(\varphi^{-1}(x)\right)^{(q-1) / q}}{\phi_{k}\left(\varphi^{-1}(x)\right)} d x \\
& =\int_{s_{1}}^{s_{2}} \frac{\left(u^{\prime}(t)\right)^{(q-1) / q}}{\phi_{k}\left(u^{\prime}(t)\right)}\left(\bar{\varphi}\left(u^{\prime}(t)\right)\right)^{\prime} d t \\
& =\int_{s_{1}}^{s_{2}} \frac{f\left(t, u(t), u^{\prime}(t)\right)}{\phi_{k}\left(u^{\prime}(t)\right)}\left(u^{\prime}(t)\right)^{(q-1) / q} d t \\
& \leq \int_{s_{1}}^{s_{2}} w(t)\left(u^{\prime}(t)\right)^{(q-1) / q} d t .
\end{aligned}
$$

Then the Hölder's inequality implies

$$
\int_{\varphi\left(r_{k}\right)}^{\varphi(K)} \frac{\left(\varphi^{-1}(x)\right)^{(q-1) / q}}{\phi_{k}\left(\varphi^{-1}(x)\right)} d x \leq\|w\|_{J_{k}, q}\left(\int_{s_{1}}^{s_{2}} u^{\prime}(t) d t\right)^{q-1} \underline{q} \leq w \|_{J_{k}, q} \eta_{k}^{(q-1) / q}
$$


where $\eta_{k}$ is defined by definition 2.3. This contradict to (4). If $u^{\prime}(\bar{t})<-K$, by a similar argument, we obtain a contradiction too. Therefore, $\left|u^{\prime}(t)\right| \leq K$ holds for $t \in[0, T]$.

To verify that condition (2) is fulfilled, we can proved in a similar way to the step 4 in Theorem 8 of [11].

Finally, we prove the solution of $\left(P^{*}\right)$ satisfies the anti-periodic boundary condition. It is suffices to prove that

$$
\begin{aligned}
& \alpha(0) \leq-u(T) \leq \beta(0), \\
& \alpha(T) \leq u(T)-u^{\prime}(0)-u^{\prime}(T) \leq \beta(T) .
\end{aligned}
$$

We will prove the first inequality of (6), by contradiction, if $\alpha(0)>-u(T)$, by the fact $u \leq \beta$, then $\alpha(0)>-u(T) \geq-\beta(T) \quad, \quad$ which contradicts to $\alpha(0)+\beta(T)=0$. The second inequality in (6) can be proved similarly. Hence, we obtain $u(0)=-u(T)$.

Let the first inequality of (7) be not satisfied, as a consequence, we have $u(T)=\alpha(T)$ and $u^{\prime}(0)+u^{\prime}(T)>0$. By $\alpha(T)+\beta(0)=0$, we have $u(0)=\beta(0)$. Together with $\alpha \leq u \leq \beta$, we can verify that $u^{\prime}(T) \leq D_{-} \alpha(T)$ and $u^{\prime}(0) \leq D_{+} \beta(0)$. Hence

$$
0<u^{\prime}(0)+u^{\prime}(T) \leq D_{-} \alpha(T)+D_{+} \beta(0) \leq 0,
$$

which is a contradiction. The second inequality of (7) can be proved similarly. Therefore, we have $u^{\prime}(0)+u^{\prime}(T)=0$.

The proof is completed.

Remark. If, instead of the implicit conditions (2), we consider the following ones

$$
I_{k}\left(u\left(t_{k}\right), u\right)=M_{k}\left(u\left(t_{k}^{+}\right), u\right)=0, k=0, \cdots, p .
$$

Assume that there exists a pair of coupled lower and upper solutions which satisfy the conditions in Definition 2.1 but assuming

$$
I_{k}\left(\alpha\left(t_{k}\right), \alpha\right) \leq 0 \leq M_{k}\left(\alpha\left(t_{k}^{+}\right), \alpha\right) .
$$

and the reversed conditions in $\beta$, together with

$\odot$ For all $k=0, \cdots, p$, functions $I_{k}: R \times C_{P}^{0}([0, T]) \rightarrow R$ are continuous and the functions $I_{k}\left(\alpha\left(t_{k}\right), \cdot\right)$ and $I_{k}\left(\beta\left(t_{k}\right), \cdot\right)$ are monotone.

$\odot$ For all $k=0, \cdots, p$, functions

$M_{k}: R \times C_{P}^{0}([0, T]) \rightarrow R$ are continuous and the functions $M_{k}\left(\alpha\left(t_{k}^{+}\right), \cdot\right)$ and $M_{k}\left(\beta\left(t_{k}^{+}\right), \cdot\right)$ are monotone.

As a consequence, we can deduce similar existence results to the ones obtained in Theorem 3.3. And we note that Remark 11 in [4], the conditions $I_{k}\left(\beta\left(t_{k}\right), \cdot\right)$ are non-decreasing and $M_{k}\left(\beta\left(t_{k}^{+}\right), \cdot\right)$ are non-increasing can be replaced by monotone.

\section{Conclusions}

In this paper, we mainly discuss the existence of solutions of anti-periodic boundary value problems for impulsive differential equations with $\varphi$-Laplacian operator. To give the existence results for the problem (1)-(3), we first introduce a pair of coupled lower and upper solutions (Definition 1) of the problem (1)-(3). Then, under the approach of coupled upper and lower solutions together with Nagumo condition (Definition 3), we deduce that there exists at least one solution of the anti-periodic boundary value problems (1)-(3) lying between a pair of coupled lower and upper solutions by the auxiliary non homogeneous impulsive Dirichlet problem.

\section{Acknowledgments}

The work was partially supported by NNSF of China Grants No.11461021, NNSF of Guangxi Grants No. 2014GXNSFAA118028, the Scientific Research Foundation of Guangxi Education Department No. KY2015YB306, Guangxi Colleges and Universities Key Laboratory of Symbolic Computation and Engineering Data Processing, and the Scientific Research Project of Hezhou University Nos. 2015ZZZK16, 2016HZXYSX07.

The authors also would like to thank the anonymous reviewer for their valuable comments and suggestions which improved the quality of the paper.

\section{References}

[1] D. Bainov, V. Covachev, Impulsive Differential Equations With a Small Parameter, World Scientific, Singapore, 1994.

[2] M. Benchohra, J. Henderson, S. K. Ntouyas, Impulsive Differential Equations and Inclusions, Hindawi Publishing Corparation, New York, 2006.

[3] V. Lakshmikantham, D. D. Bainov, P.S. Simeonov, Theory of Impulsive Differential Equations, World Scientific, Singapore, 1989.

[4] A. Cabada, J. Tomecek, Extremal solutions for nonlinear functional $\varphi$-Laplacian impulsive equations, Nonlinear Anal. 67 (2007) 827-841.

[5] W. Ding, M. A. Han, J. Mi, Periodic boundary value problem for the second order impulsive functional equations, Comput. Math. Appl. 50 (2005) 491-507.

[6] A. Cabada, B. Thompson, Nonlinear second-order equations with functional implicit impulses and nonlinear functional boundary conditions, Nonlinear Anal. 74 (2011) 7198-7209.

[7] E. N. Dancer. On the Dirichlet problem for weakly non-linear elliptic partial differential equations. Proc. Roy. Soc. Edinburgh Sect. A, 76 (1977) 283-300.

[8] A. Cabada, An overview of the lower and upper solutions method with nonlinear boundary value conditions, Bound. Value Probl. Art. ID 893753 (2011) 1-18.

[9] H. Kleinert, A. Chervyakov, Functional determinants from Wronski Green function, J. Math. Phys. 40 (1999) 6044-6051. 
[10] M. Wang, A. Cabada, J. J. Nieto, Monotone method for nonlinear second order periodic boundary value problems with Carath'eodory functions, Ann. Polon. Math. 58(3) (1993) 221-235.

[11] A. Cabada, D. R. Vivero, Existence and uniqueness of solutions of higher-order antiperiodic dynamic equations, Adv. Difference Equ. 4 (2004) 291-310.

[12] X. Guo, L. Lu, Z. Liu, BVPs for higher-order integro-differential equations with $\phi$-Laplacian and functional boundary conditions, Adv. Differ. Equa. 2014:285 (2014) 1-13.

[13] P. Jebelean, C. Şerban, Boundary value problems for discontinuous perturbations of singular $\phi$-Laplacian operator. J. Math. Anal. Appl. 431(1) (2015) 662-681.
[14] H. Okochi, On the existence of periodic solutions to nonlinear abstract parabolic equations, J. Math. Soc. Japan 40(3) (1988) 541-553.

[15] F. Delvos, L. Knoche, Lacunary interpolation by anti-periodic trigonometric polynomials, BIT 39 (1999) 439-450.

[16] P. Wang, W. Wang, Anti-periodic boundary value problem for first order impulsive delay difference equations, Adv. Differ. Equ. 2015(1) (2015) 1-13.

[17] T. Zhang, Y. Li, Global exponential stability and existence of anti-periodic solutions to impulsive Cohen-Grossberg neural networks on time scales, Topol. Methods Nonlinear Anal. 45(2) (2015) 363-384. 